\title{
Self-gravitating axially symmetric disks in general-relativistic rotation
}

\author{
Janusz Karkowski, Wojciech Kulczycki, Patryk Mach, Edward Malec, Andrzej Odrzywołek, and Michał Piróg \\ Instytut Fizyki im. Mariana Smoluchowskiego, Uniwersytet Jagielloński, Eojasiewicza 11, 30-348 Kraków, Poland
}

We integrate numerically axially symmetric stationary Einstein equations describing selfgravitating disks around spinless black holes. The numerical scheme is based on a method developed by Shibata, but contains important new ingredients. We derive a new general-relativistic Keplerian rotation law for self-gravitating disks around spinning black holes. Former results concerning rotation around spinless black holes emerge in the limit of a vanishing spin parameter. These rotation curves might be used for the description of rotating stars, after appropriate modification around the symmetry axis. They can be applied to the description of compact torus-black hole configurations, including active galactic nuclei or products of coalescences of two neutron stars.

PACS numbers: 04.20.-q, 04.25.Nx, 04.40.Nr, 95.30.Sf

\section{INTRODUCTION}

Stationary axially symmetric systems of rotating polytropic fluid share two common properties both in general relativity and in Newtonian gravity. They need additional specification - the so-called rotation curve, that tells particles of fluid how to rotate. They are freeboundary elliptic systems - the shape of rotating fluids cannot be set a priori, but constitutes a part of the solution. In Newtonian gravity the Poincaré-Wavre theorem allows one to define explicitly a rich family of allowed simple rotations $\Omega=\Omega(\varpi)$, where $\Omega$ is the angular velocity, and $\varpi$ denotes the distance from the rotation axis. In contrast to that, the general-relativistic laws of rotation had been rather poorly recognised. The classical rotation curves used in general relativity [1, 2] are essentially variants of uniform rotation. Keplerian rotations are common in rotating astrophysical systems, but in Newtonian limits of known rotation curves [1/5 the Keplerian rotation law is obtained only approximately.

We proposed in [6] a general-relativistic differential rotation law $j=j(\Omega)$, where $j$ denotes the specific angular momentum, capable to describe a stationary system consisting of a self-gravitating disk circulating around a spinless (or spinning) black hole. Its most striking characteristic is that the nonrelativistic limit exactly coincides with Newtonian angular velocities $\Omega=w / \varpi^{\lambda}$ $(0 \leq \lambda \leq 2)$. In particular the Keplerian rotation law $\Omega \propto 1 / \varpi^{3 / 2}$ belongs to the Newtonian limit of this general-relativistic rotation curve. The rotation law 6 . has been recently investigated up to the first order of the post-Newtonian approximation (1PN), for a spinless black hole [7.

One of the two main aims of this paper is the generalization of results obtained in [6]. We shall derive a general-relativistic Keplerian rotation law, that might be more convenient for the description of tori moving around spinning black holes.

From the formal point of view the admissible rotation law can be specified freely both in general relativity, as a function $j=j(\Omega)$, and in Newtonian gravity, as $\Omega=\Omega(\varpi)$. However not for every rotation law the cou- pled system of Einstein-Euler or Poisson-Euler equations admits solutions for a reasonably wide range of solution parameters. Since sufficiently general exact existence results are at present only available for the Newtonian case 8, one has to resort to a numerical evidence of existence or absence of solutions. As we will argue in the forthcoming sections, this procedure actually yields practical information on the form of robust rotation laws.

Our second main objective is to show that compact systems consisting of a spinless black hole and a torus in the general-relativistic rotation [6] (that depends on only one parameter) can be described numerically in the full Einstein theory. We describe the black hole-toroid system within the puncture framework implemented in 9], but there are important modifications. One of them is that adopted rotation laws lead to a set of highly nonlinear algebraic equations that need to be solved during iterations. We imposed in a different way than Hachisu [10] one of construction conditions for numerical toroids, that significantly improved convergence of iterations. We test our code on the Kerr solution, which in turn led to implementations of some boundary conditions that might be different.

Recent investigation shows that there are reasons to conjecture that stellar mass black hole-torus systems emerge as end products of binary neutron stars or black hole-neutron star mergers [11] and references therein], associated with some detections of gravitational waves. Moreover, fluid tori in these systems do self-gravitate and rotate with the Keplerian velocity [12-15] around a spinning black hole.

The present paper gives a complete description of the formalism and obtained results. In the next section we briefly describe the Einstein equations and the equations of hydrodynamics, explaining the rotation law of [6]. Relevant information on the numerical scheme is given in Sec. III. Section IV reports results of numerical tests. In particular, we perform standard convergence tests and recover numerical results of Shibata 9 . We discuss in Sec. $\mathrm{V}$ the status of rotation parameters in the rotation law of 6; it appears that the rotation law is rather rigid and there is only one parameter that is free and another that emerges as a part of the numerical solu- 
tion. In the subsequent section we show profiles of a few toroids for different systems - with constant angular momentum density, the Keplerian angular rotation and for systems with tori in (almost) uniform linear velocity rotation. Section VII is dedicated to the derivation of a new rotation curve that is expected to be efficient in describing systems with spinning black holes. The result of 6] appears in the limit of the vanishing spin of the black hole. Section VIII shows explicitly that the Poincaré-Wavre (Newtonian) property of the angular velocity - that the angular velocity does not depend on the height above the symmetry plane - can be robustly broken in general relativity. Finally, we summarize obtained results and outline prospects for further related research.

We investigated the whole interval of $a / m \in[-0.9,0.9]$ (where $a$ and $m$ are the spin and mass parameters of the black hole, respectively), that includes cases shown to be of interest $a / m \in[0.6,0.9]$ in simulations of coalescences of neutron stars [11, 16.

\section{EQUATIONS}

We assume a stationary metric of the form

$$
\begin{aligned}
d s^{2}=- & \alpha^{2} d t^{2}+r^{2} \sin ^{2} \theta \psi^{4}(d \varphi+\beta d t)^{2} \\
& +\psi^{4} e^{2 q}\left(d r^{2}+r^{2} d \theta^{2}\right)
\end{aligned}
$$

Here $t$ is the time coordinate, and $r, \theta, \varphi$ are spherical coordinates. The gravitational constant $G=1$ and the speed of light $c=1$. We assume axial symmetry and employ the stress-momentum tensor

$$
T^{\alpha \beta}=\rho h u^{\alpha} u^{\beta}+p g^{\alpha \beta},
$$

where $\rho$ is the baryonic rest-mass density, $h$ is the specific enthalpy, and $p$ is the pressure. Metric functions $\alpha(r, \theta)$, $\psi(r, \theta), q(r, \theta)$ and $\beta(r, \theta)$ in (1) depend on $r$ and $\theta$ only.

The following method can be applied to any barotropic equation of state but we will deal with polytropes $p(\rho)=$ $K \rho^{\gamma}$. Then one has the specific enthalpy

$$
h(\rho)=1+\frac{\gamma p}{(\gamma-1) \rho} .
$$

The 4-velocity $\left(u^{\alpha}\right)=\left(u^{t}, 0,0, u^{\varphi}\right)$ is normalized, $g_{\alpha \beta} u^{\alpha} u^{\beta}=-1$. The coordinate angular velocity reads

$$
\Omega=\frac{u^{\varphi}}{u^{t}}
$$

It is well known that general-relativistic Euler equations are solvable under the integrability condition that $j \equiv j(\Omega)$ depends only on the angular velocity [1, 2]. Within the fluid region, the Euler equations $\nabla_{\mu} T^{\mu \nu}=0$ can be integrated, yielding

$$
\int h u_{\varphi} d \Omega+\frac{h}{u^{t}}=C_{1}
$$

or

$$
\int u^{t} u_{\varphi} d \Omega+\ln \left(\frac{h}{u^{t}}\right)=C_{2} .
$$

We choose the latter form, and define the angular momentum per unit inertial mass $\rho h$ [17.

$$
j \equiv u_{\varphi} u^{t}
$$

In 6] we have had the rotation law

$$
j(\Omega) \equiv \frac{\tilde{w}^{1-\delta} \Omega^{\delta}}{1-\kappa \tilde{w}^{1-\delta} \Omega^{1+\delta}+\Psi} ;
$$

here $\Psi$ is of the order of the binding energy per unit baryonic mass. This law followed from an "educated guesswork," in which three elements played equally important roles: the rotation law should have the right (monomial) Newtonian limit, it should yield the right $1 \mathrm{PN}$ correction to the angular velocity, and the Bernoulli equation should have a unique form. The last demand was ensured by imposing the condition that a massless disk of dust exactly satisfies the Einstein-Bernoulli equations. The full reasoning is described in [6].

Simple rescaling

$$
w^{1-\delta}=\frac{\tilde{w}^{1-\delta}}{1+\Psi}
$$

transforms (5) into

$$
j(\Omega) \equiv \frac{w^{1-\delta} \Omega^{\delta}}{1-\kappa w^{1-\delta} \Omega^{1+\delta}}=\left(-\kappa \Omega+w^{\delta-1} \Omega^{-\delta}\right)^{-1},
$$

where $w, \delta$, and $\kappa=(1-3 \delta) /(1+\delta)$ are parameters. Thus the rotation law reads

$$
j(\Omega) \equiv\left(-\kappa \Omega+w^{\delta-1} \Omega^{-\delta}\right)^{-1},
$$

where $w, \delta$, and $\kappa$ are parameters. The rotation curves $\Omega(r, z)$ ought to be recovered from the Eq. (4), which can be written as

$$
j(\Omega)=\frac{V^{2}}{(\Omega+\beta)\left(1-V^{2}\right)},
$$

where the square of the linear velocity reads

$$
V^{2}=r^{2} \sin ^{2} \theta(\Omega+\beta)^{2} \frac{\psi^{4}}{\alpha^{2}},
$$

and $j(\Omega)$ is defined by (6). The integro-algebraic Bernoulli equation (3) is given by a simple algebraic form 6

$$
h \alpha \sqrt{1-V^{2}}\left(1-\kappa w^{1-\delta} \Omega^{1+\delta}\right)^{-\frac{1}{(1+\delta) \kappa}}=C .
$$

The central black hole is surrounded by a minimal twosurface $S_{\mathrm{BH}}$ located at $r=r_{\mathrm{s}}$ in the puncture method [18, on a fixed hypersurface of constant time. Its area 
defines the irreducible mass $M_{\mathrm{irr}}=\sqrt{\frac{A_{\mathrm{H}}}{16 \pi}}$ and its angular momentum $J_{\mathrm{H}}$ follows from the Komar expression

$$
J_{\mathrm{H}}=\frac{1}{4} \int_{0}^{\pi / 2} \frac{r^{4} \psi^{6}}{\alpha} \partial_{r} \beta \sin ^{3} \theta d \theta .
$$

The angular momentum is prescribed rigidly on the event horizon $S_{\mathrm{BH}}$-it is given by data taken from the Kerr solution (with two parameters, mass $m$ and the spin parameter $a=J_{\mathrm{H}} / m$ ) and it is independent of the content of mass in a torus. The mass of the black hole is then defined as

$$
M_{\mathrm{BH}}=M_{\mathrm{irr}} \sqrt{1+\frac{J_{\mathrm{H}}^{2}}{4 M_{\mathrm{irr}}^{4}}} .
$$

Another possible choice of the black hole mass is in terms of the circumferential radius $r_{\mathrm{C}} \equiv r \sin \theta \psi^{2}$ of $S_{\mathrm{BH}}$ at the symmetry plane $\theta=\pi / 2: \quad M_{\mathrm{C}}=r_{\mathrm{C}} / 2$. We observed that in our numerical calculations $M_{\mathrm{C}}$ and $M_{\mathrm{BH}}$ differ by significantly less than $1 \%$; this is consistent with findings of Shibata 9 . The asymptotic mass $M_{\mathrm{ADM}}$ is defined as in 9. We decided to define the mass of tori by $m_{\mathrm{T}} \equiv M_{\mathrm{ADM}}-M_{\mathrm{BH}}$. The characteristic feature of this construction is that the metric of the whole spacetime coincides with the Kerr metric in the limit of $m_{\mathrm{T}} \rightarrow 0$.

Let $K_{i j}$ denote the extrinsic curvature of the $t=$ const hypersurface. Define the conformal extrinsic curvature $\hat{K}_{i j}$ as $\hat{K}_{i j}=\psi^{2} K_{i j}$. The only nonzero component $\beta$ of the shift vector is split as $\beta=\beta_{\mathrm{K}}+\beta_{\mathrm{T}}$, where $\beta_{\mathrm{K}}$ and $\beta_{\mathrm{T}}$ are determined as follows. The nonvanishing components of $\hat{K}_{i j}$ can be written in the form

$$
\begin{gathered}
\hat{K}_{r \varphi}=\frac{H_{\mathrm{E}} \sin ^{2} \theta}{r^{2}}+\frac{\psi^{6}}{2 \alpha} r^{2} \sin ^{2} \theta \partial_{r} \beta_{\mathrm{T}}, \\
\hat{K}_{\theta \varphi}=\frac{H_{\mathrm{F}} \sin \theta}{r}+\frac{\psi^{6}}{2 \alpha} r^{2} \sin ^{2} \theta \partial_{\theta} \beta_{\mathrm{T}} .
\end{gathered}
$$

As in [9], we choose the functions $H_{\mathrm{E}}$ and $H_{\mathrm{F}}$ to be expressed by the formulas obtained for the Kerr metric of mass $m$ and the spin parameter $a$, written in the form (1). In explicit terms they read [18]

$$
\begin{gathered}
H_{\mathrm{E}}=\frac{m a\left[\left(r_{\mathrm{K}}^{2}-a^{2}\right) \Sigma_{\mathrm{K}}+2 r_{\mathrm{K}}^{2}\left(r_{\mathrm{K}}^{2}+a^{2}\right)\right]}{\Sigma_{\mathrm{K}}^{2}}, \\
H_{\mathrm{F}}=-\frac{2 m a^{3} r_{\mathrm{K}} \sqrt{r_{\mathrm{K}}^{2}-2 m r_{\mathrm{K}}+a^{2}} \cos \theta \sin ^{2} \theta}{\Sigma_{\mathrm{K}}^{2}},
\end{gathered}
$$

where

$$
r_{\mathrm{K}}=r\left(1+\frac{m}{r}+\frac{m^{2}-a^{2}}{4 r^{2}}\right)
$$

and

$$
\Sigma_{\mathrm{K}}=r_{\mathrm{K}}^{2}+a^{2} \cos ^{2} \theta .
$$

It follows that for the Kerr metric, one has

$$
\hat{K}_{r \varphi}=\frac{H_{\mathrm{E}} \sin ^{2} \theta}{r^{2}}
$$

and

$$
\hat{K}_{\theta \varphi}=\frac{H_{\mathrm{F}} \sin \theta}{r} .
$$

In the presence of the torus $\beta_{\mathrm{K}}$ has to be computed from the relation

$$
\frac{\partial \beta_{\mathrm{K}}}{\partial r}=\frac{2 H_{\mathrm{E}} \alpha}{r^{4} \psi^{6}}
$$

In what follows we proceed with the puncture method as implemented in 9]. Define $\Phi=\alpha \psi$ and assume the puncture at $r=0$. Define $r_{\mathrm{s}}=\frac{1}{2} \sqrt{m^{2}-a^{2}}$, and

$$
\psi=\left(1+\frac{r_{\mathrm{s}}}{r}\right) e^{\phi}, \quad \Phi=\left(1-\frac{r_{\mathrm{s}}}{r}\right) e^{-\phi} B .
$$

The surface $r=r_{\mathrm{s}}$ is an apparent horizon.

Einstein equations can be written as

$$
\begin{aligned}
{\left[\partial_{r r}+\frac{1}{r} \partial_{r}+\frac{1}{r^{2}} \partial_{\theta \theta}\right] q } & =S_{q}, \\
{\left[\partial_{r r}+\frac{2 r}{r^{2}-r_{\mathrm{s}}^{2}} \partial_{r}+\frac{1}{r^{2}} \partial_{\theta \theta}+\frac{\cot \theta}{r^{2}} \partial_{\theta}\right] \phi } & =S_{\phi}, \\
{\left[\partial_{r r}+\frac{3 r^{2}+r_{\mathrm{s}}^{2}}{r\left(r^{2}-r_{\mathrm{s}}^{2}\right)} \partial_{r}+\frac{1}{r^{2}} \partial_{\theta \theta}+\frac{2 \cot \theta}{r^{2}} \partial_{\theta}\right] B } & =S_{B}, \\
{\left[\partial_{r r}+\frac{4 r^{2}-8 r_{\mathrm{s}} r+2 r_{\mathrm{s}}^{2}}{r\left(r^{2}-r_{\mathrm{s}}^{2}\right)} \partial_{r}+\frac{1}{r^{2}} \partial_{\theta \theta}+\frac{3 \cot \theta}{r^{2}} \partial_{\theta}\right] \beta_{\mathrm{T}} } & =S_{\beta_{\mathrm{T}}},
\end{aligned}
$$


where source terms $S_{\phi}, S_{B}, S_{\beta_{\mathrm{T}}}, S_{q}$ are

$$
\begin{aligned}
& S_{q}=-8 \pi e^{2 q}\left(\psi^{4} p-\frac{\rho h u_{\varphi}^{2}}{r^{2} \sin ^{2} \theta}\right)+\frac{3 A^{2}}{\psi^{8}}+2\left[\frac{r-r_{\mathrm{s}}}{r\left(r+r_{\mathrm{s}}\right)} \partial_{r}+\frac{\cot \theta}{r^{2}} \partial_{\theta}\right] b+\left[\frac{8 r_{\mathrm{s}}}{r^{2}-r_{\mathrm{s}}^{2}}+4 \partial_{r}(b-\phi)\right] \partial_{r} \phi \\
& +\frac{4}{r^{2}} \partial_{\theta} \phi \partial_{\theta}(b-\phi), \\
& S_{\phi}=-2 \pi e^{2 q} \psi^{4}\left[\rho_{\mathrm{H}}-p+\frac{\rho h u_{\varphi}^{2}}{\psi^{4} r^{2} \sin ^{2} \theta}\right]-\frac{A^{2}}{\psi^{8}}-\partial_{r} \phi \partial_{r} b-\frac{1}{r^{2}} \partial_{\theta} \phi \partial_{\theta} b-\frac{1}{2}\left[\frac{r-r_{\mathrm{s}}}{r\left(r+r_{\mathrm{s}}\right)} \partial_{r} b+\frac{\cot \theta}{r^{2}} \partial_{\theta} b\right], \\
& S_{B}=16 \pi B e^{2 q} \psi^{4} p, \\
& S_{\beta_{\mathrm{T}}}=\frac{16 \pi \alpha e^{2 q} J}{r^{2} \sin ^{2} \theta}-8 \partial_{r} \phi \partial_{r} \beta_{\mathrm{T}}+\partial_{r} b \partial_{r} \beta_{\mathrm{T}}-8 \frac{\partial_{\theta} \phi \partial_{\theta} \beta_{\mathrm{T}}}{r^{2}}+\frac{\partial_{\theta} b \partial_{\theta} \beta_{\mathrm{T}}}{r^{2}}
\end{aligned}
$$

and

$$
\begin{gathered}
A^{2}=\frac{\hat{K}_{r \varphi}^{2}}{r^{2} \sin ^{2} \theta}+\frac{\hat{K}_{\theta \varphi}^{2}}{r^{4} \sin ^{2} \theta}, \\
\rho_{\mathrm{H}}=\rho h\left(\alpha u^{t}\right)^{2}-p, \\
J=\rho h \alpha u^{t} u_{\varphi}, \\
B=e^{b} .
\end{gathered}
$$

In the rest of the paper we always assume that $\Omega>0$. Corotating disks have $a>0$, while counterrotating disks have negative spins: $a<0$.

\section{DESCRIPTION OF NUMERICS}

The core of our numerical method is close to that described in 9. Main modifications introduced in our scheme are due to the implementation of a different rotation law which requires solving Eq. (7). The Kerr solution plays an essential role in the presented model. It appears formally in the limit $m_{\mathrm{T}} \rightarrow 0$. It is important to stress that the assumed rotation law (6) actually admits solutions with an arbitrarily small mass. The latter property is by no means obvious; it is for instance absent for the rigid rotation [19. Moreover, the Kerr metric is the only analytic solution that is available for testing the correctness of our numerical code. Consequently, we implemented some boundary conditions in the way that yields the Kerr solution with the best accuracy in the absence of the torus. Finally, there is a technical difference in treating Eq. (12d).

The solutions are found iteratively, using a fixed-point method. Equations 12 are solved with respect to functions $\phi, B, \beta_{\mathrm{T}}$, and $q$, respectively. In each iteration Eqs. (11) is integrated yielding $\beta_{\mathrm{K}}$. The specific enthalpy is computed from the Bernoulli Eq. (8). Our prescription of the rotation law in the form $j=j(\Omega)$ requires also solving Eq. (7) with respect to $\Omega$.
The baryonic density $\rho$ is computed from the specific enthalpy as

$$
\rho=\left[\frac{\gamma-1}{K \gamma}(h-1)\right]^{\frac{1}{\gamma-1}} .
$$

In each iteration we set the polytropic constant $K$ so that the maximum of the density is equal to an a priori specified value $\rho_{\max }$; this is essentially the old idea of Hachisu [10, to which we added a new element. We observed that the convergence of the fixed point method significantly improves, i.e., solutions can be obtained for a much wider range of parameters, if the maximum of the enthalpy is sought within the whole volume of the disk, and not only at the equatorial plane. On the other hand, in all cases investigated so far, the final solution is characterized by the maximum of the specific enthalpy occurring at the plane $\theta=\pi / 2$. The constants $w$ and $C$ appearing in the rotation law and Eq. (8) are hard to control, and they are not specified a priori. Instead, we assume the values of the inner and outer equatorial radii of the disk ( $r_{1}$ and $r_{2}$, respectively). The values of $w$ and $C$ are obtained in each iteration from Eqs. (7) and (8) taken at points with the coordinates $(r, \theta)$ equal to $\left(r_{1}, \pi / 2\right)$ and $\left(r_{2}, \pi / 2\right)$. They constitute a set of four algebraic equations for four unknowns: $w, C$, and the values of $\Omega$ at $\left(r_{1}, \pi / 2\right)$ and $\left(r_{2}, \pi / 2\right)$. Fortunately, simple substitutions are possible; they lead effectively to an algebraic set of two equations, which we solve using a standard Newton-Raphson method.

Our numerical grid spans the region $r_{\mathrm{s}} \leq r \leq r_{\infty}$, $0 \leq \theta \leq \pi / 2$, where $r_{\infty}$ is finite, but large: $r_{\infty} \gg r_{2}$. We assume equatorial symmetry. This implies that $\partial_{\theta} \phi=$ $\partial_{\theta} B=\partial_{\theta} \beta_{\mathrm{T}}=\partial_{\theta} q=0$ at $\theta=\pi / 2$. On the axis we assume regularity conditions, that is $\partial_{\theta} \phi=\partial_{\theta} B=\partial_{\theta} \beta_{\mathrm{T}}=$ 0 at $\theta=0$. We also put $q=0$ at $\theta=0$, which is required by the local flatness of the metric. The boundary conditions at $r=r_{\mathrm{s}}$ follow from the puncture construction. We require that $\partial_{r} \phi=\partial_{r} B=\partial_{r} \beta_{\mathrm{T}}=\partial_{r} q=0$ at $r=r_{\mathrm{s}}$. It is observed in [9] that Eq. 12d) admits a more stringent condition at $r=r_{\mathrm{s}}$. We follow [9] and require $\beta_{\mathrm{T}}=O\left[\left(r-r_{\mathrm{s}}\right)^{4}\right]$, or equivalently $\partial_{r} \beta_{\mathrm{T}}=\partial_{r r} \beta_{\mathrm{T}}=\partial_{r r r} \beta_{\mathrm{T}}=0$ at $r=r_{\mathrm{s}}$. To some 
extent this choice is arbitrary and it is connected with the freedom of defining the splitting of the shift vector $\beta=\beta_{\mathrm{T}}+\beta_{\mathrm{K}}$, but it has consequences in the definition and properties of the angular momentum of the black hole.

The conditions at the outer boundary follow from the multipole expansion and the conditions of asymptotic flatness. We have

$$
\begin{aligned}
\phi & \sim \frac{M_{1}}{2 r}, \quad B \sim 1-\frac{B_{1}}{r^{2}}, \\
\beta_{\mathrm{T}} & \sim-\frac{2 J_{1}}{r^{3}}, \quad q \sim \frac{q_{1} \sin ^{2} \theta}{r^{2}},
\end{aligned}
$$

as $r \rightarrow \infty$, where the constants $M_{1}, B_{1}, J_{1}$ and $q_{1}$ satisfy

$$
\begin{gathered}
M_{1}=-2 \int_{r_{\mathrm{s}}}^{\infty}\left(r^{2}-r_{\mathrm{s}}^{2}\right) d r \int_{0}^{\pi / 2} \sin \theta d \theta S_{\phi}, \\
B_{1}=\frac{2}{\pi} \int_{r_{\mathrm{s}}}^{\infty} d r \frac{\left(r^{2}-r_{\mathrm{s}}^{2}\right)^{2}}{r} \int_{0}^{\pi / 2} d \theta \sin ^{2} \theta S_{B}, \\
J_{1}=4 \pi \int_{r_{\mathrm{s}}}^{\infty} r^{2} d r \int_{0}^{\pi / 2} \sin \theta d \theta \rho \alpha u^{t} \psi^{6} e^{2 q} h u_{\varphi}, \\
q_{1}=\frac{2}{\pi} \int_{r_{\mathrm{s}}}^{\infty} d r r^{3} \int_{0}^{\pi / 2} d \theta \cos (2 \theta) S_{q} \\
-\frac{4}{\pi} r_{\mathrm{s}}^{2} \int_{0}^{\pi / 2} d \theta \cos (2 \theta) q\left(r_{\mathrm{s}}, \theta\right) .
\end{gathered}
$$

Note a misprint in the boundary condition for $B$ in 9 .

In our implementation the grid points can be distributed almost freely, both in radial and angular directions, but we require existence of grid points at the boundaries, i.e., for $r=r_{\mathrm{s}}, \theta=0, \theta=\pi / 2$ and $r=r_{\infty}$. In practice we distribute the grid points in the radial direction according to

$$
r_{i}=r_{\mathrm{s}}+\frac{f^{i-1}-1}{f-1} \Delta r, \quad i=1,2, \ldots, N_{r}
$$

where $f$ and $\Delta r$ are constants. This choice is similar to the one used in 9 . In the angular direction we either set an equidistant grid

$$
\theta_{j}=(j-1) \Delta \theta, \quad j=1, \ldots, N_{\theta},
$$

where $\Delta \theta=\pi /\left[2\left(N_{\theta}-1\right)\right]$, or a grid with almost equally spaced values of $\mu=\cos \theta$, i.e.,

$$
\theta_{j}= \begin{cases}0, & j=1, \\ \arccos \left[1+\left(\frac{3}{2}-j\right) \Delta \mu\right], & j=2, \ldots, N_{\theta}-1, \\ \frac{\pi}{2}, & j=N_{\theta},\end{cases}
$$

where $\Delta \mu=1 /\left(N_{\theta}-2\right)$.

Equations 12 are discretized using second order (3point) difference formulas. The implementation of the boundary conditions is more subtle. In each iteration we set the values of $\phi, B, \beta_{\mathrm{T}}$ and $q$ at the boundary grid nodes, assuring that appropriate boundary conditions are satisfied. At the outer boundary, i.e., for $r=r_{\infty}$, we set the values of $\phi, B, \beta_{\mathrm{T}}$ and $q$ as given by Eqs. (14) and (15)-18). At the axis $(\theta=0)$, equator $(\theta=\pi / 2)$, and the horizon $\left(r=r_{\mathrm{s}}\right)$, the boundary conditions generally amounts to a requirement that an appropriate first derivative vanishes. We express this derivative using 3-, 4- or 6-point formulas, and set the boundary values appropriately. These boundary values are treated as fixed in the process of solving Eq. (12) for $\phi, B, \beta_{\mathrm{T}}$, and $q$ in the bulk of the grid. In other words, in each iteration, Eqs. 12 are solved as if they constituted a Dirichlet boundary problem. This approach seems to be essential for the convergence properties of the iterative fixed-point scheme. The only exception from this rule is our treatment of the boundary condition for $\beta_{\mathrm{T}}$ at the horizon. In the latter case the equations expressing the conditions $\partial_{r} \beta_{\mathrm{T}}=\partial_{r r} \beta_{\mathrm{T}}=\partial_{r r r} \beta_{\mathrm{T}}=0$ at $r=r_{\mathrm{s}}$ are solved together with Eq. (12d) using 6-point finite difference formulae. Other boundary conditions are implemented using 3-point formulas. A 4-point finite difference formula is used only for the condition $\partial_{r} \phi=0$ at $r=r_{\mathrm{s}}$. We would like to stress that this choice yielded the best accuracy in the test consisting in recovering the Kerr solution in the absence of the torus. On the other hand, it is not optimal with respect to the computation of the $R_{\text {con }}$ parameter, which we describe in Sec. IV A.

All integrals (15)-18 are computed using standard trapezoid formulae in each iteration. Trapezoid formulas are also used in the integration of Eq. (11) for $\beta_{\mathrm{K}}$. Following 9 , we assume that $\beta_{\mathrm{K}}=0$ at $r=r_{\infty}$, which is understood as an approximation of the boundary condition $\beta_{\mathrm{K}} \rightarrow 0$ for $r \rightarrow \infty$.

Most of the computing time is spent on solving the discretized versions of Eqs. 12. We exploit the banded matrix structure of these four equations, and solve them performing standard $L U$ matrix decomposition [20] using LAPACK [21. A technical change as compared to the formulation of [9] is that we have moved terms depending on the derivatives of $\phi$ and $b$ to the right-hand side of Eq. (12d). As a result the coefficients of the operators on the left-hand sides of Eq. 12 do not change during iterations, and it is possible to perform the decomposition of the matrices once only. Equation (7) is solved in each iteration with respect to $\Omega$, using a NewtonRaphson method. We search for the solution for $\Omega$ in a bounded region containing the torus.

Choosing appropriate initial data is an inherent problem of all iterative methods for obtaining relativistic figures of equilibrium. Rotation law (6) admits starting from the Kerr data, i.e., there exist an acceptable solution of Eq. (8) for the Kerr metric, except for the case with $a=0$, and $\kappa=3$. We obtain the solutions of the latter case by first obtaining a solution for $\kappa=3$ and a small, but nonzero $a$. This solution is then used as a starting point to obtain the final solution for $\kappa=3$ and 
$a=0$

In addition to the parameters of the rotation law $[\delta$ and $\kappa$ in case of the rotation law [6] ], the solution is specified by the following six parameters: the mass $m$ and the spin $a$ parameters of the black hole; the inner and outer coordinate radii of the disk at the equatorial plane: $r_{1}$ and $r_{2}$, respectively; the maximum of the baryonic density within the disk $\rho_{\max }$; the polytropic exponent $\gamma$. We assume in what follows $m=1$ and $\gamma=4 / 3$. All length related quantities $\left(r_{1}, r_{2}\right.$ and circumferential radii) shall be expressed in terms of $m$.

We should comment here on terminology. As we have pointed above, in the construction of Shibata 9 the angular momentum of the black hole is defined a priori in terms of parameters $a$ and $m$ : the "dressed" angular momentum is the same as the naked one, $J_{\mathrm{BH}}=a m$. On the other hand the "dressed" mass of a black hole $M_{\mathrm{BH}}$ is different from the mass parameter $m$. Thus $J_{\mathrm{BH}} / M_{\mathrm{BH}} \neq a$; the notion of the spin parameter $a$ would become ambiguous. In order to avoid misunderstanding, when we use the term spin parameter $a$, we always mean that $J_{\mathrm{BH}}=a m$.

\section{TESTING NUMERICAL CODES}

We shall provide below a short discussion of the accuracy of our numerical codes. We begin, in the first subsection, with elements of standard numerical analysis. Then we shall compare our results on two metric functions with those of [9].

\section{A. Numerical tests}

From the mathematical point of view, a solution for a given rotation law [with one free parameter, e.g., (6) with fixed $\kappa$ ], is expected to be defined by "physical" parameters: $m, a, r_{1}, r_{2}, \rho_{\max }$. This solution might not be unique, in principle. However, numerical solutions depend on several additional parameters. The most obvious is the grid size $N_{r} \times N_{\theta}$ and its spacing. Our mesh is rectangular in the radial and angular variables. The inner edge in radius is by construction always positioned at the apparent horizon $r_{\mathrm{s}}$, but the outer edge radius $r_{\infty}$ provides another numerical parameter. It might influence asymptotic expansions used to define outer boundary conditions, see Eqs. (14)-18. Noteworthy, it is directly related to the grid spacing $\Delta r$ near the horizon (19). Less obvious numerical errors come from the surface-capturing scheme, which does not allow one to define the free boundary with the accuracy better than the size of a cell. In particular, in our implementation inner and outer radii $r_{1}, r_{2}$ are pushed upward to nearest equatorial mesh points and the aliasing error is inevitable. Finally, the "fixed-point" in practice is never reached. The convergence is limited by a numerical noise, albeit it is of a tiny amplitude. The forthcoming analysis will quantify dependence of the results on variations of $N_{r}, N_{\theta}, r_{\infty}$ or $\Delta r$ and the number of iterations. Grids as small as $N_{r} \times N_{\theta}=17 \times 9$ and as big as $N_{r} \times N_{\theta}=8193 \times 257$ were used in testing procedures. The lowest resolution yields results that are too inaccurate but the numerical solution still exists. This demonstrates robustness and stability of the numerical code.

In the next three subsections we put $a=0.6, r_{1}=8$, $r_{2}=20, \rho_{\max }=4 \times 10^{-4}$ and use the rotation law (6) with $\delta=-1 / 3$ and $\kappa=3$. We applied the geometric variant of 19$)$, i.e., with $\Delta r=(f-1) r_{\mathrm{s}}$; this implies $r_{i}=r_{\mathrm{s}} f^{i-1}$ with $f=1.0113622034834149$. That facilitates the grid doubling 1 . The radius $r_{\infty}$ was kept constant by squaring $f$ or taking the square root of $f$ when the grid was halved or doubled relative to $N_{r}=511$, respectively. We used the grid equidistant in $\cos \theta$, in tests concerning the angular resolution. The grid resolutions $N_{r} \times N_{\theta}$ are defined as integer powers of 2 minus 1, to keep them odd. The machine precision $\epsilon \simeq 2.22 \times 10^{-16}$ was shown in Figs. 14 for reference. We use the maximum norm $L^{\infty}$ to quantify errors. We measure the difference between the values of metric functions and the enthalpy in two subsequent iterations. One can use other matrix norms that would give (as we have checked) values larger or smaller by a few orders of magnitude. On the other hand error ratios and line slopes shown in Figs. 1,4 are invariant.

\section{Fixed point noise}

A typical behavior during numerical iterations (Figs. 1) consists of three stages: (1) a short (a few hundred iterations) divergent stage with the maximum mass density occurring outside the symmetry plane $\theta=\pi / 2$; (2) a stage characterized by an exponential convergence $\left(10^{3}-10^{4}\right.$ iterations); (3) a steady stage with the error dominated by the numerical noise. With the increased grid size two first stages are longer both with respect to the number of iterations and the computational time. The amplitude of the numerical noise is increased as well. Fortunately, in all investigated cases the magnitude of the noise is small and of no significance. In practice, the errors associated with other factors, which we describe below, are always larger. Consequently, in most cases it suffices to fix the number of iterations at a level of a few thousands.

\section{Doubling the grid: Increasing $N_{r}$ and $N_{\theta}$ with fixed $r_{\infty}$}

By doubling the grid we simultaneously reduce two sources of errors: (1) the discretization error; (2) the aliasing error. Typically, doubling the radial grid gives an

\footnotetext{
1 By doubling we understand adding new grid points between adjacent points of the old grid.
} 

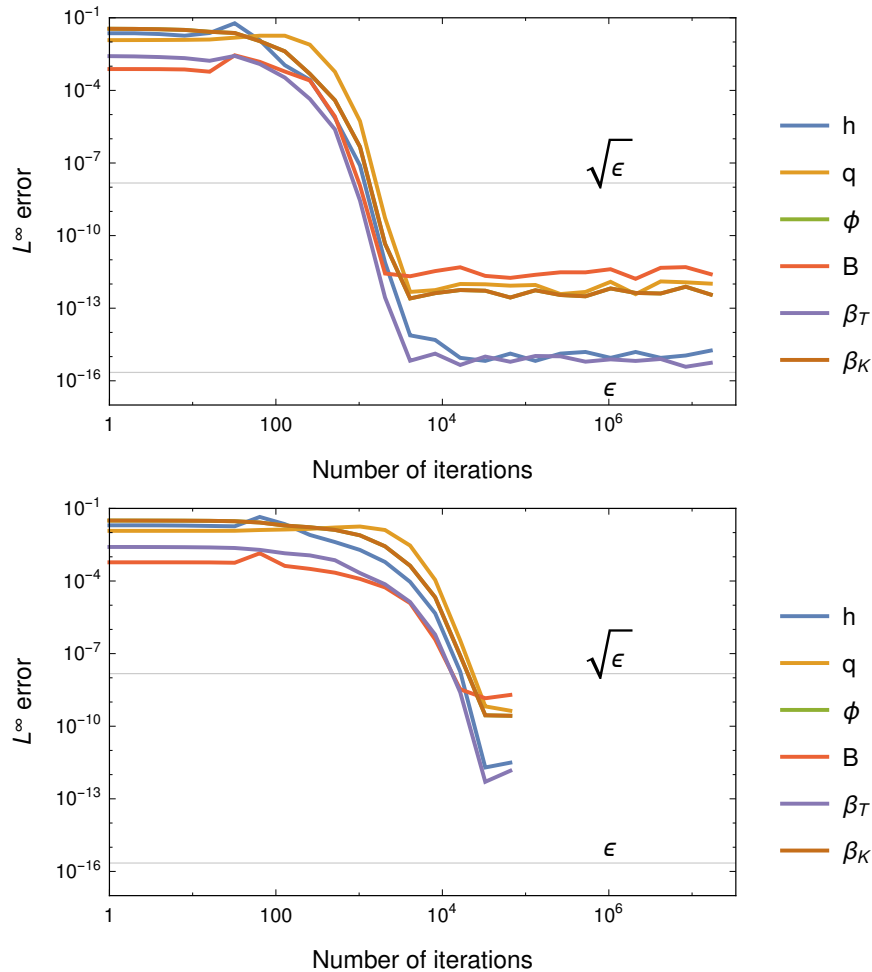

$-\phi$

$-\mathrm{B}$

$-\beta_{T}$

$-\beta_{K}$

FIG. 1. Typical behavior of the $L^{\infty}$ error measured with respect to the preceding iteration for two extreme cases with the grids $N_{r} \times N_{\theta}=2049 \times 257$ (the bottom diagram) and $N_{r} \times N_{\theta}=65 \times 33$ (the upper diagram).

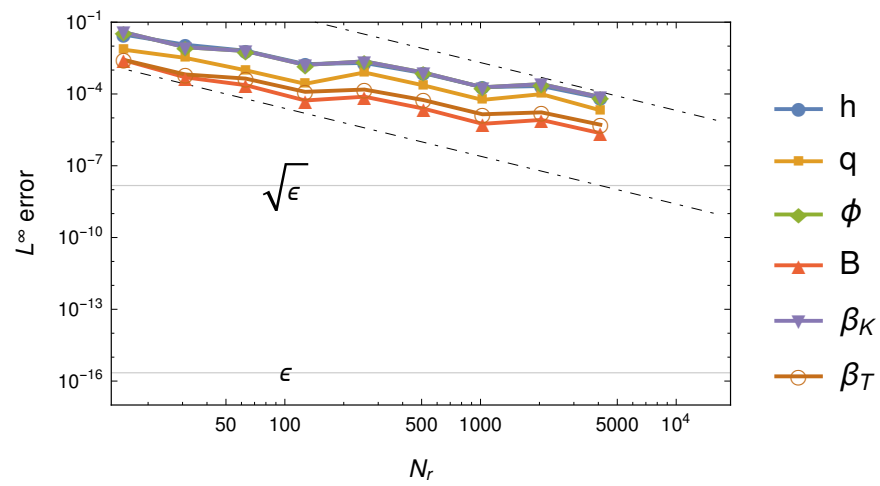

FIG. 2. Typical behavior of the $L^{\infty}$ error with the increase of the radial grid resolution. Here $r_{\infty}$ is constant. Lowest resolution $N_{r} \times N_{\theta}=17 \times 17$ was doubled in radial direction nine times until reference model $N_{r} \times N_{\theta}=8193 \times 17$ (not shown) was obtained.

almost quadratic convergence (Fig. 2), while doubling the angular grid (free of an aliasing error) yields convergence that is exactly quadratic (cf. Fig. 3).

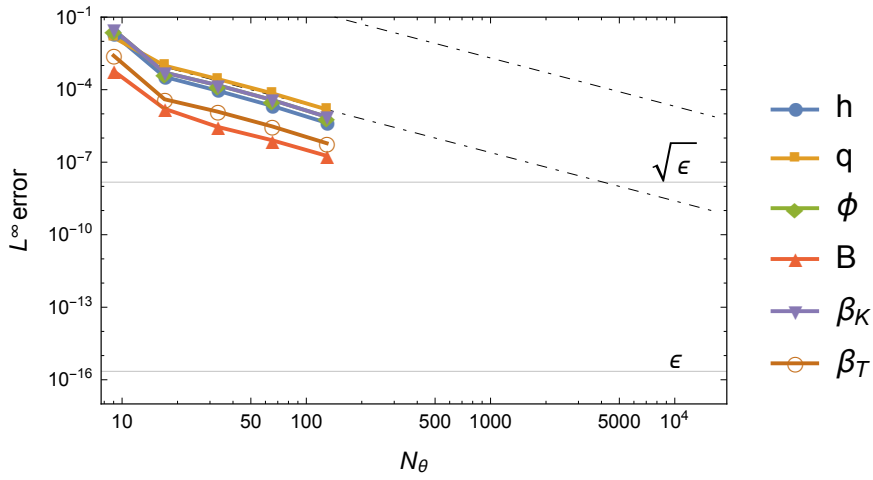

FIG. 3. The same as in Fig. 2, but with the increased angular resolution. The grid grows from $N_{r} \times N_{\theta}=1025 \times 9$ to $N_{r} \times N_{\theta}=1025 \times 257$.

\section{Extending the grid: Increasing $N_{r}$ with fixed $f$ and $\Delta r$}

The extension of the outer grid edge $r_{\infty}$ has a rather moderate impact on the global error, which decreases linearly with the increase of $r_{\infty}$ (see Fig. 4).

$$
\text { 4. } R_{\text {con }} \text { test }
$$

In order to disentangle the discretization and aliasing errors, we employ the test of Shibata (see the Appendix B in (9) for grid-aligned tori. One can compute the value:

$$
R_{\mathrm{con}}=\frac{Q_{4}-Q_{1}}{Q_{2}-Q_{1}}
$$

where $Q_{1}, Q_{2}, Q_{4}$ denotes values obtained at some grid node with the use of the original radial grid, a grid with every second node removed and another grid obtained from the former by repeating the removal operation. For the interpolation of the $n$th order one could expect $R_{\text {con }}=2^{n}+1$, i.e., $R_{\text {con }}=5$ for the quadratic

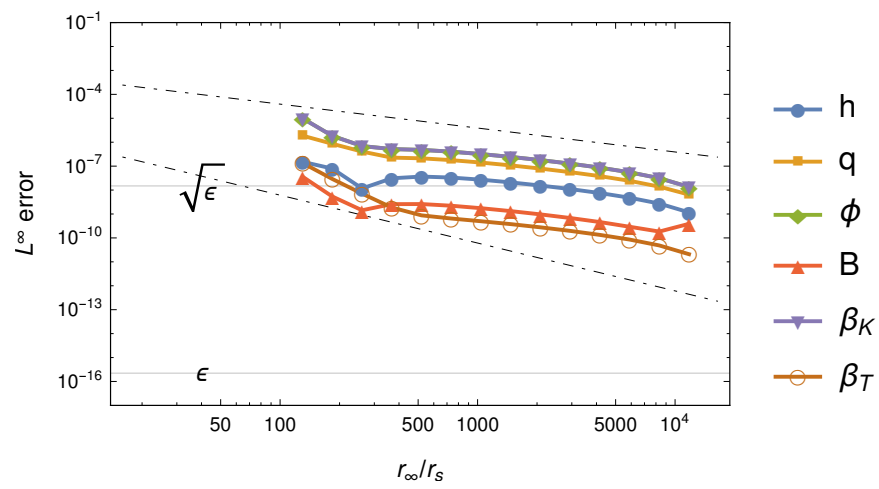

FIG. 4. Typical behavior of the $L^{\infty}$ error with the increase of the $r_{\infty}$. Radial grid was extended from $N_{r} \times N_{\theta}=513 \times 65$ to $N_{r} \times N_{\theta}=961 \times 65$, while keeping $f$ and $\Delta r$ constant in (19). 
interpolation/discretization. Unfortunately, results that have been obtained are rather inconclusive. For Shibata's test model and for one of our models (a central black hole with the spin parameter $a=0.9$ and the rotation law (39) with $a=0.9$ and $\kappa=3$ ) we get in fact $R_{\text {con }} \simeq 5 \pm 0.05$, for all metric functions with the exception of $q$ and a few grid nodes in the vicinity of the horizon. In this test we implement boundary conditions at the horizon for $\phi$ and $q$ using 3- and 4-point finite difference formulas, respectively.

On the other hand, for our model with the rotation law (22), and with the same spin parameter of the central black hole, $a=0.9$, the situation becomes confusing. We get $R_{\text {con }} \simeq 5 \pm 1$ at best, with a large variation for some metric functions (see Fig. 5). Clarification of these issues requires further testing, but we are not convinced that the tests based on the $R_{\text {con }}$ parameter are necessarily useful. It is important to stress, that any value of $R_{\text {con }}>1$ indicates the convergence. There are values of $R_{\text {con }} \gg 5$ (Fig. 5. lower panel) that indicate convergence better than expected. This might be surprising, but it is possible. For example, if one of the metric function is well-approximated by a quadratic polynomial, the 2nd order scheme could yield almost exact solution of the discretized system instantly.
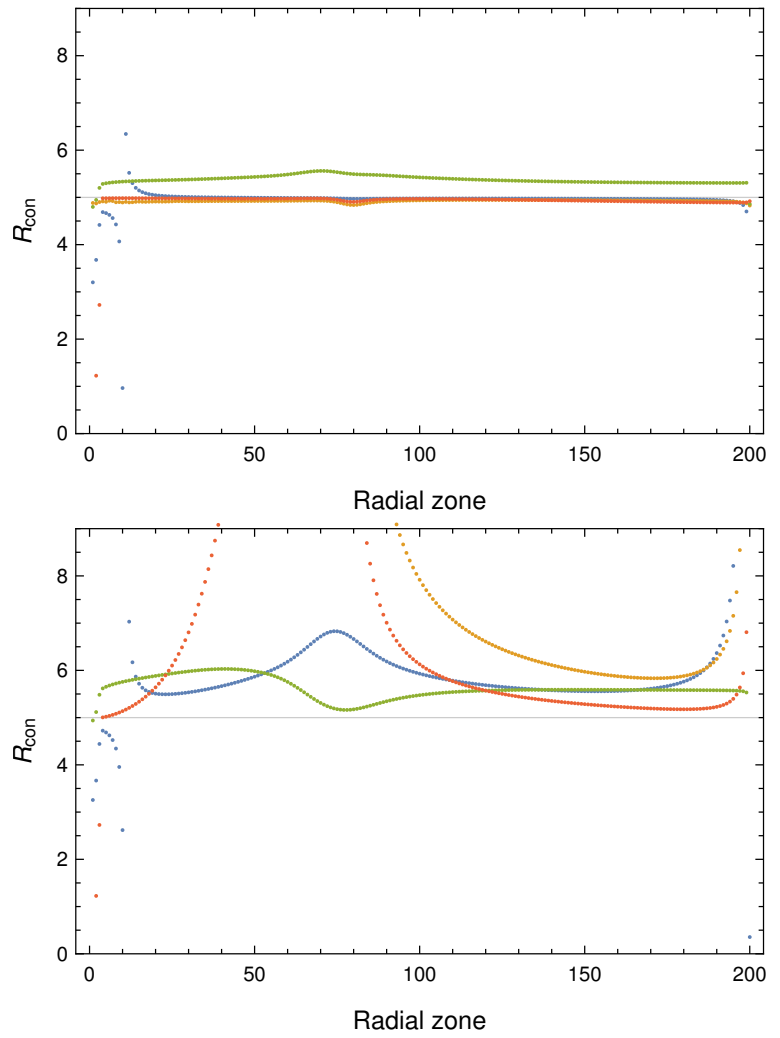

FIG. 5. Results for $R_{\text {con }}$ tests. In the upper part of the figure the black hole has $a=0.9$ and the torus rotates according to (39), where $\kappa=3$. In the lower part the black hole has $a=0.9$ and the torus rotates according to 22 .

\section{Conclusions}

Judging from the contribution to the $L^{\infty}$ norm of the error, its main sources can be ordered as follows. The radial resolution (including aliasing), and the angular resolution contribute $\left(10^{-3}-10^{-5}\right)$ and $\left(10^{-5}-10^{-7}\right)$ to the budget error, respectively. Further contributions come from the radial grid extension $\left(10^{-8}-10^{-10}\right)$ and the fixed-point noise error $\left(10^{-11}-10^{-16}\right)$.

Apart from the convergence tests described above, we have also recovered the Kerr metric for the vacuum case, i.e., without the torus. The code successfully passed all these tests.
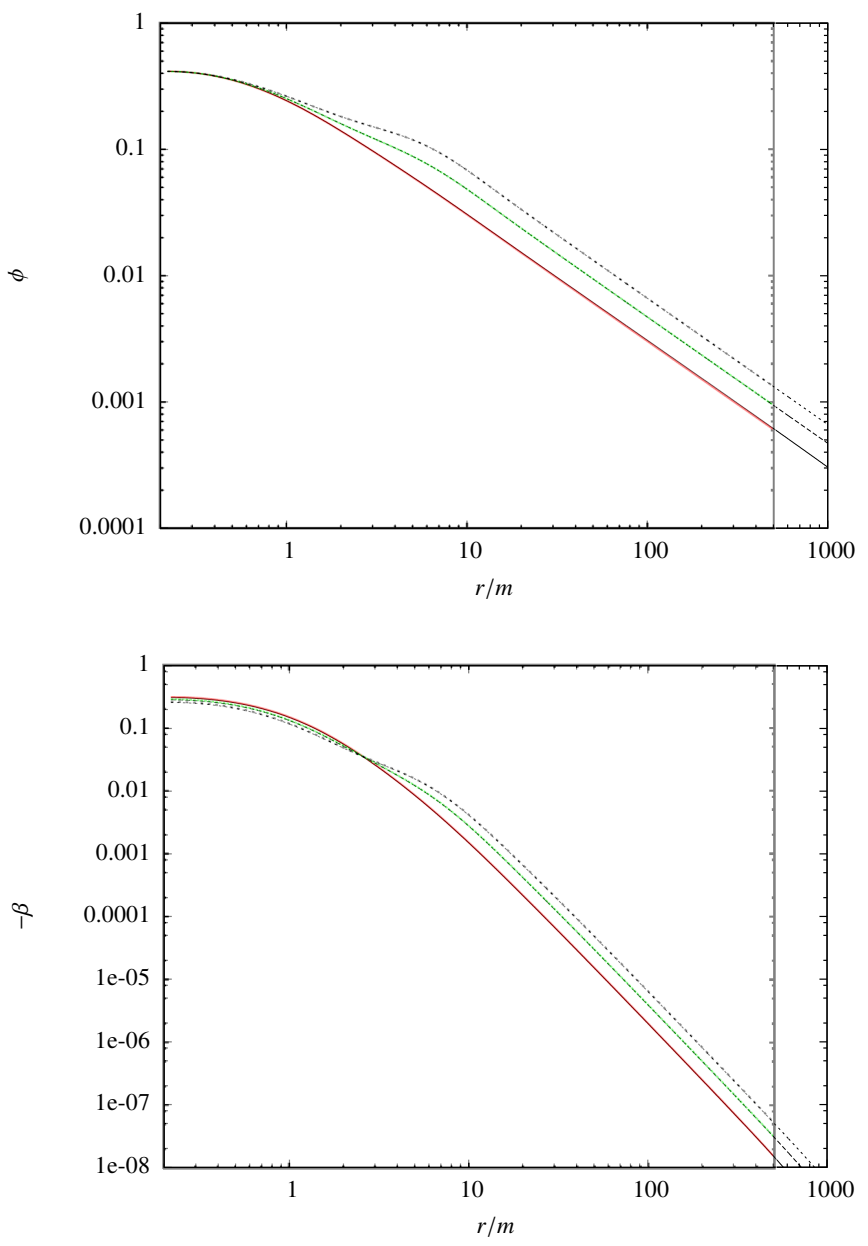

FIG. 6. Functions $\phi$ and $-\beta$ in the equatorial plane for the configuration with the spin parameter of the black hole $a=0.9, r_{1}=1.5, r_{2}=20.0$, for the cases $M_{*} / m=$ $0.043, \rho_{\max }=0.475 \times 10^{-4}$ (solid line),$M_{*} / m=0.400$, $\rho_{\max }=3.128 \times 10^{-4}$ (broken line) and $M_{*} / m=0.800$, $\rho_{\max }=4.936 \times 10^{-4}$ (dashed-dotted line). Inset in the two figures (with the abscissa extending up to $r / m=500$ ) are the corresponding diagrams taken from Fig. 4 of 9 . 


\section{B. Rotation with constant $h u_{\varphi}$}

We do several calculations using the same parameters and the same rotation curve - defined by the constancy of the angular momentum density $j_{\mathrm{Sh}}=h u_{\varphi}$-as in [9]. Solutions have been found for parameter sets (spin parameter $a$, inner $r_{1}$ and outer $r_{2}$ coordinate radii, respectively): (i) $(0.9,1.5,20)$ with $M_{*}=0.043,0.4,0.8$ and (ii) $(0.1,5,20)$ with $M_{*}=0.093,0.405,0.835$. The quantity $M_{*}$ defined as in 9 is a kind of a baryonic mass of the torus. Figures 6 and 7 portray two families of solutions of two metric functions $\phi$ and $\beta$ in the plane $\theta=\pi / 2$. The results presented in Figs. 6 and 7 agree quite well with the results presented in Fig. 4 of $[9$. There are two exceptions - the plots of $\phi$ and $-\beta\left(M_{*}=0.405\right)$ in Fig.
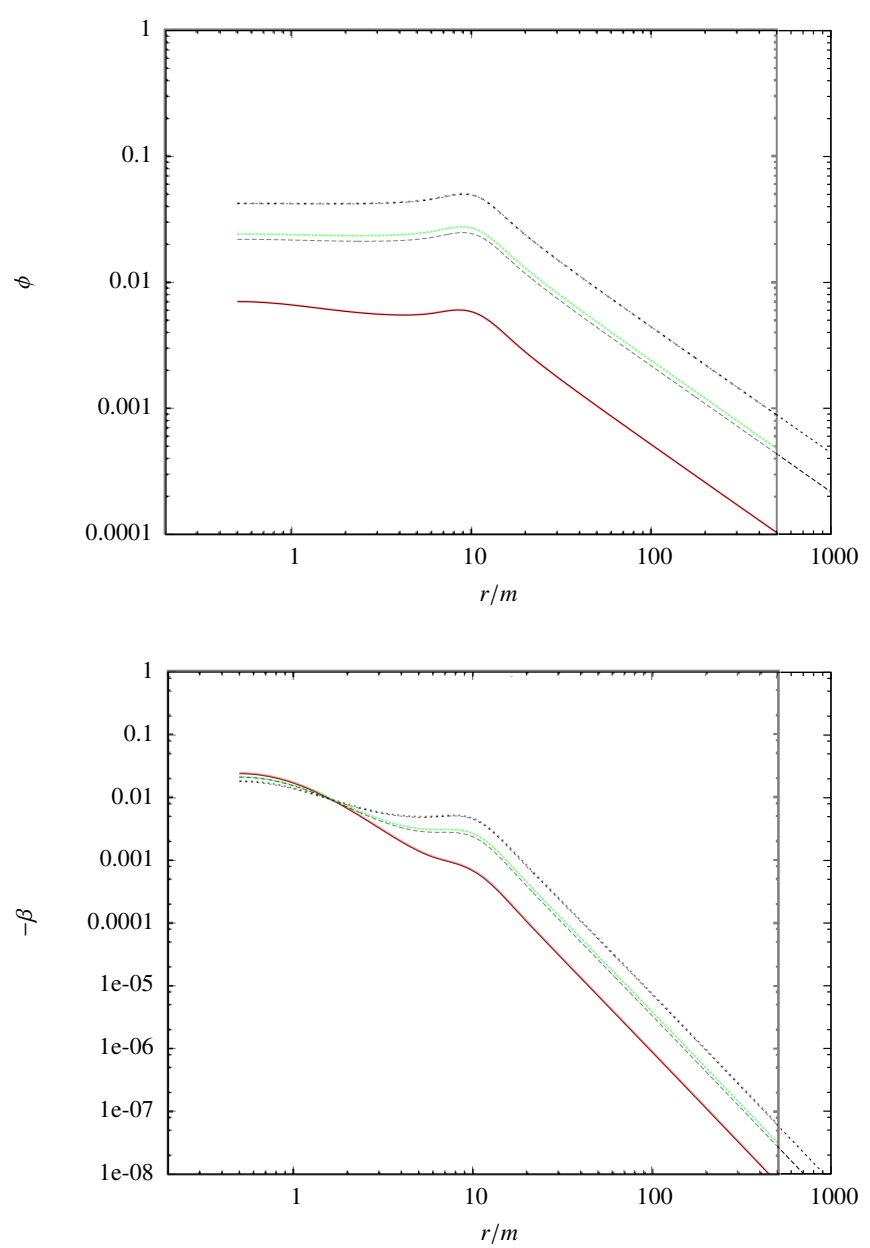

FIG. 7. Functions $\phi$ and $-\beta$ in the equatorial plane for the configuration with the spin parameter of the black hole $a=0.1, r_{1}=5.0, r_{2}=20.0$, for the cases $M_{*} / m=$ $0.093, \rho_{\max }=0.445 \times 10^{-4}$ (solid line),$M_{*} / m=0.405$, $\rho_{\max }=1.704 \times 10^{-4}$ (broken line) and $M_{*} / m=0.835$, $\rho_{\max }=3.083 \times 10^{-4}$ (dashed-dotted line). Inset in the two figures (with the abscissa extending up to $r / m=500$ ) are the corresponding diagrams taken from 9 .
7 reveal a difference.

Our solutions presented in Figs. 6 and 7 were computed on the numerical grids characterized by $\Delta r=r_{\mathrm{s}} / 50, f=$ 1.01, $N_{r}=802, N_{\theta}=102$. This yields $r_{\infty} \approx 5800 r_{\mathrm{s}}$. The grid nodes were distributed in the angular direction according to Eq. 20.

\section{THE COEFFICIENT $\kappa$ IN THE KEPLERIAN ROTATION LAW OF [6]}

The rotation law (6) seems to have two free parameters: $\kappa$ and $w$. The parameter $\delta$ is dictated by the Newtonian limit

$$
\Omega_{0}=\frac{w}{\varpi^{\frac{2}{1-\delta}}}
$$

and for the Keplerian rotation one has to choose $\delta=$ $-1 / 3$. Then the formula $\kappa=(1-3 \delta) /(1+\delta)$, required by the first post-Newtonian expansion, yields $\kappa=3$ and

$$
j(\Omega) \equiv\left(-3 \Omega+w^{-4 / 3} \Omega^{1 / 3}\right)^{-1} .
$$

Numerical results, reported below, suggest that in fact the general-relativistic Keplerian rotation is best described by (22). Clearly the parameter $w$ is not free-its value constitutes a part of a solution.

Solutions presented in this and the following sections were computed on the numerical grids characterized by $\Delta r=r_{\mathrm{s}} / 50, f=1.01, N_{r}=802, N_{\theta}=102$. The grid nodes were distributed in the angular direction according to Eq. 21.

We performed a large number of numerical calculations for spin-less central black holes in order to find out whether $\kappa=3$ is the best choice for the Keplerian rotation. They are shown in Table I] From these and other calculations, we infer that with the increase of mass, while keeping the inner boundary close to the innermost stable circular orbit (ISCO), the interval $\left(\kappa_{\min }, \kappa_{\max }\right)$ for which we were able to find solutions, shifts downwards: lower and upper bounds for $\kappa$ go down. We can conclude

TABLE I. Rotation around a spinless black hole of the mass $m=1$. The first 2 columns give the innermost and outermost radii of tori, the third describes maximal values of the baryonic mass density, the fourth gives the disk's mass (with $\kappa=3$ ) and the final two columns show the minimal and maximal of values of $\kappa$ for which solutions were found.

\begin{tabular}{cccccc}
\hline \hline$r_{1}$ & $r_{2}$ & $\rho_{\max }$ & $m_{\mathrm{T}}$ & $\kappa_{\min }$ & $\kappa_{\max }$ \\
\hline 5.04 & 20.1 & $0.2 \times 10^{-4}$ & $4.5 \times 10^{-3}$ & 3.0 & 4.9 \\
5.04 & 20.1 & $1.0 \times 10^{-4}$ & $7.8 \times 10^{-2}$ & 2.9 & 4.8 \\
5.04 & 20.1 & $5.0 \times 10^{-4}$ & 1.1 & 1.5 & 4.0 \\
5.04 & 10.1 & $0.2 \times 10^{-3}$ & $7.2 \times 10^{-3}$ & 3.0 & 5.2 \\
5.04 & 10.1 & $1.0 \times 10^{-3}$ & $1.1 \times 10^{-1}$ & 2.7 & 5.0 \\
5.04 & 10.1 & $4.0 \times 10^{-3}$ & 1.0 & 0.8 & 3.9 \\
\hline \hline
\end{tabular}


TABLE II. The spin of central black holes is given in the first row. The second row displays values of the parameter $\kappa$ for which the mass gap disappears. In all examples below the inner and outer radii are $r_{1}=8$ and $r_{2}=20$, respectively

\begin{tabular}{cccccc}
\hline \hline$a$ & -0.9 & -0.8 & -0.7 & -0.6 & -0.5 \\
$\kappa$ & 3.91 & 3.81 & 3.71 & 3.61 & 3.512 \\
$a$ & -0.4 & -0.3 & -0.2 & -0.1 & -0.05 \\
$\kappa$ & 3.409 & 3.308 & 3.206 & 3.103 & 3.052 \\
\hline \hline
\end{tabular}

also, that for the general-relativistic Keplerian rotation with $\delta=-1 / 3$, the choice of $\kappa=3$ is the safe choice, that always gives solutions. Furthermore, calculations with some other pairs of values $\delta, \kappa=(1-3 \delta) /(1+\delta)$ always yielded solutions (see next section). That might mean that $w$ is the only parameter in the rotation law 22 that cannot be defined a priori.

We investigated also systems with spinning black holes and tori rotating according to (22). We have always found solutions for corotation $(a>0$ and $\Omega>0$ ), assuming $\kappa=3$. The situation is different-solutions with small masses of the tori do not necessarily exist for $\kappa=3$-in the case of counterrotating systems. In fact, the value of $\kappa$, for which solutions with light tori exist, can be distinctly different from 3 . We have found a mass gap for counterrotating tori $(\Omega>0$ and $a<0)$, with $\kappa=3$ and $a \in[-0.1,-0.9]$ - tori may exist only if their mass is larger than a particular mass threshold. The existence of this mass threshold is interesting from the mathematical point of view, since it means that the space-time geometry produced by counterrotating disks with the above specified rotation law does not tend to the Kerr geometry; solutions seem to disappear when masses of toroids become too small. On the other hand it appears that this feature is not physical for the rotation curve (6) -we have found that the gap disappears for other values of $\kappa$. Table II shows such values of $\kappa$ for a selection of spins of central black holes.

\section{CHARACTERISTICS OF TORI ROTATING AROUND SPINLESS BLACK HOLES}

We investigated solutions corresponding to the rotation law (6) for $\delta \in[-0.95,0]$ and $\kappa$ given by the formula $\kappa=(1-3 \delta) /(1+\delta)$, or in its close vicinity. Below we present the following cases:

(i) constant angular momentum density $j: \delta=0, \kappa=$ 1

(ii) the Keplerian rotation $\delta=-1 / 3, \kappa=3$;

(iii) $\delta=-0.8, \kappa=17$; this gives a linear velocity that is slowly changing across the symmetry plane of the torus;

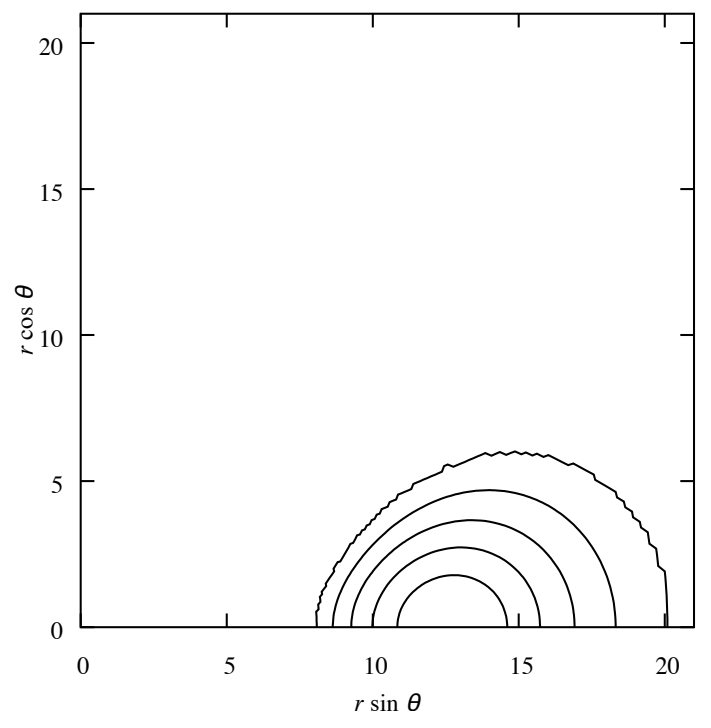

FIG. 8. Isolines of constant enthalpy $h$, for non-spinning black hole and the rotation law (6) with $\delta=0$ and $\kappa=1$. The black hole mass $M_{\mathrm{BH}}=1.0567, \rho_{\max }=3.45 \times 10^{-4}$ and $h_{\max }=2.67 \times 10^{-2}$.

(iv) $\delta=-0.95, \kappa=77$; this gives a linear velocity that varies very little on the intersection of the symmetry plane with the torus.

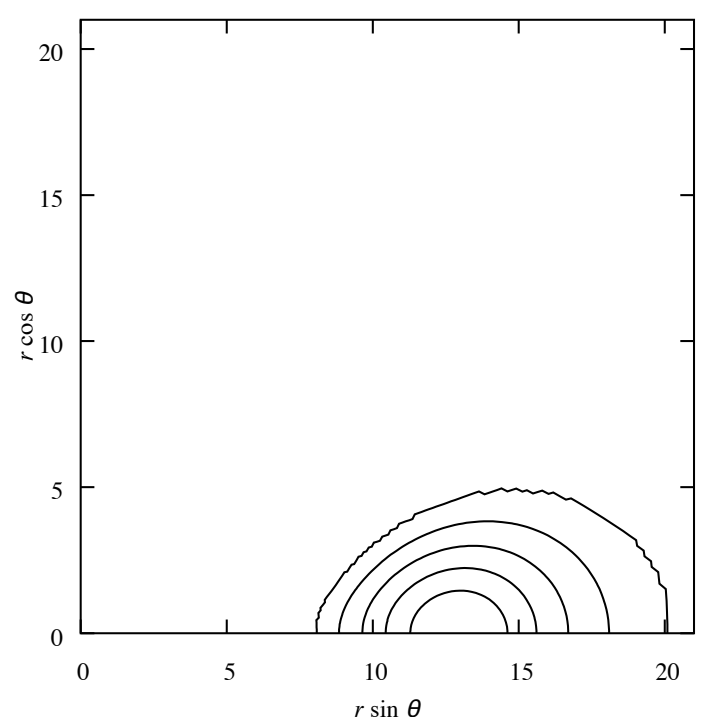

FIG. 9. Isolines of constant enthalpy $h$, for nonspinning black hole and the rotation law 22 . The black hole mass $M_{\mathrm{BH}}=1.0562, \rho_{\max }=4.60 \times 10^{-4}$ and $h_{\max }=2.31 \times 10^{-2}$. 


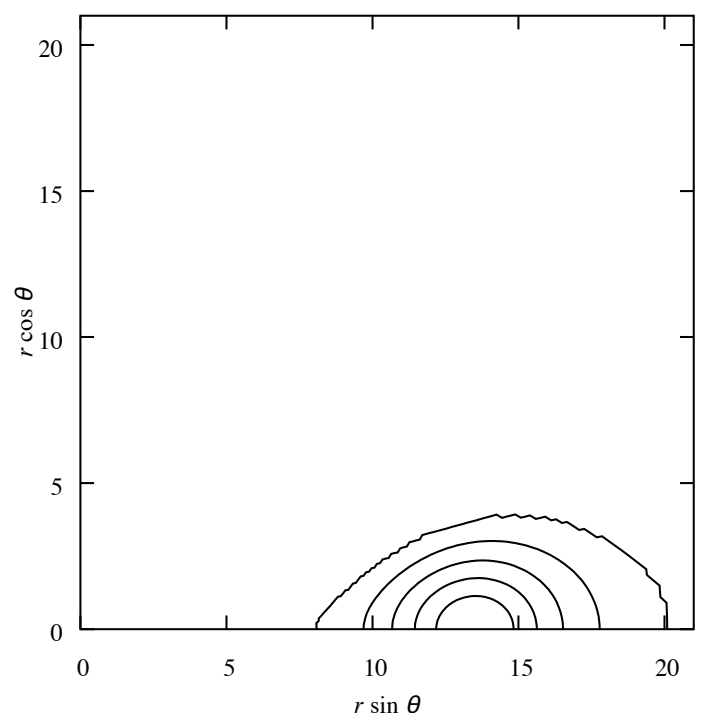

FIG. 10. Isolines of constant enthalpy $h$, for non-spinning black hole and the rotation law (6) with $\delta=-0.8$ and $\kappa=17$. The black hole mass $M_{\mathrm{BH}}=1.0542, \rho_{\max }=6.97 \times 10^{-4}$ and $h_{\max }=2.01 \times 10^{-2}$.

The case $\delta=-1$ corresponds to the constant linear velocity, but it requires a separate numerical implemen-

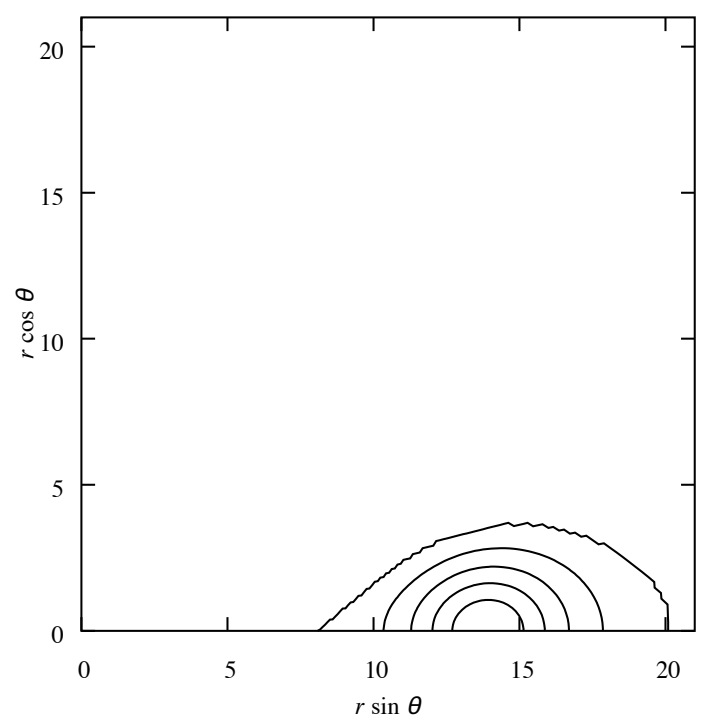

FIG. 11. Isolines of constant enthalpy $h$, for nonspinning black hole and the rotation law (6) with $\delta=-0.95$ and $\kappa=$ 77. The black hole mass $M_{\mathrm{BH}}=1.0531, \rho_{\max }=7.93 \times 10^{-4}$ and $h_{\max }=1.92 \times 10^{-2}$.

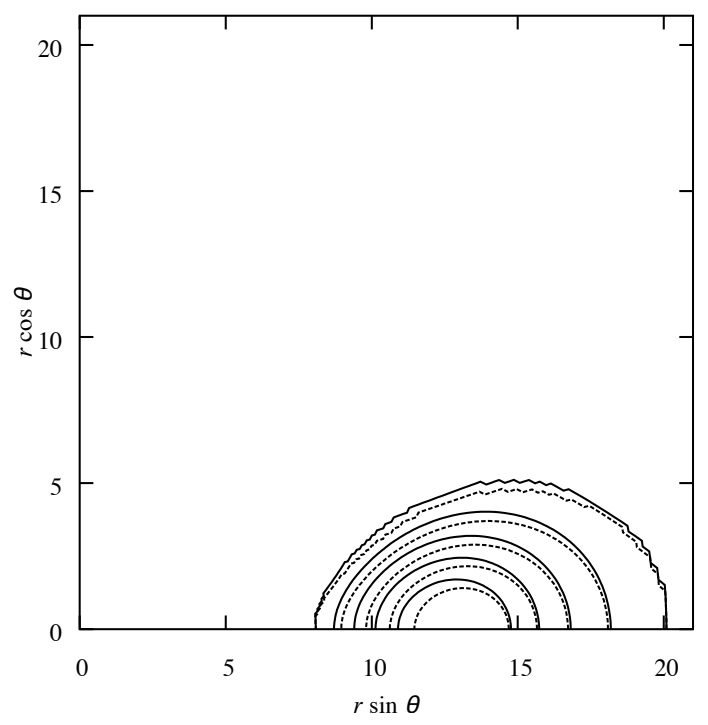

FIG. 12. Isolines of constant enthalpy $h$, for nonspinning black holes and the rotation law (22) with $\delta=-1 / 3$ for $\kappa=$ 2.5 (broken lines) and $\kappa=3.5$ (solid lines). The black hole masses are $M_{\mathrm{BH}}=1.0557(\kappa=2.5)$ and $M_{\mathrm{BH}}=1.0566(\kappa=$ 3.5). The maximal mass densities and maximal enthalpy are $\rho_{\max }=4.85 \times 10^{-4}$ and $h_{\max }=2.24 \times 10^{-2}$, and $\rho_{\max }=$ $4.37 \times 10^{-4}$ and $h_{\max }=2.38 \times 10^{-2}$, for $\kappa=2.5$ and $\kappa=3.5$ respectively.

tation of the rotation law 22 .

Solutions depicted in Figs. 8 12 were computed assuming the same asymptotic mass $M_{\mathrm{ADM}}=1.7288$, spinless black holes and the innermost and outermost coordinate radii $r_{1}=8.079$ and $r_{2}=20.09$, respectively. The masses of black holes appear to be very similar, which means that tori masses are essentially the same, $m_{\mathrm{T}}=$ $M_{\mathrm{ADM}}-M_{\mathrm{BH}} \approx 0.67$. The corresponding circumferential radii are similar, $r_{\mathrm{C}}\left(r_{1}\right) \in(9.64,9.69)$ and $r_{\mathrm{C}}\left(r_{2}\right) \in$ (21.99, 22.02). The 4 inner isolines in Figs. 811 are defined by the following formula $h_{i}=0.2(4-i)\left(h_{\max }-1\right)+1$ for $i=0,1,2,3$, while the outermost isoline corresponds to $h=1.00000001$. In Fig. 12 the isolines are defined in the same way, but $h_{\max }$ is the maximal enthalpy for the configuration with $\kappa=2.5$.

There is a number of interesting features that depend on the choice of the rotation curve.

(i) profiles of tori circularize with the increase of $\delta$ and flatten with the decrease of $\delta$-inspect Fig. 8 11. Tori area also decreases. On the other hand, keeping $\delta=-1 / 3$ and changing $\kappa$ induces smaller differences (see Fig. 12).

(ii) the maximal mass density $\rho_{\max }$ increases with the decrease of $\delta$ (see inscriptions to forthcoming figures). The increase is by a factor of two between 
the cases of the constant angular momentum density and the almost linear rotation. The dependence on the parameter $\kappa$ for fixed $\delta$ seems to be less pronounced (see Fig. 12.

(iii) the maximal enthalpy $h_{\max }$ decreases with the decrease of $\delta$ (see captions of the forthcoming figures).

\section{NEW ROTATION LAW FOR SPINNING BLACK HOLES}

We shall start with deriving the rotation law for a massless disk of dust encircling a spinning Kerr black hole in the symmetry plane. As will be shown below, this is a tedious algebraic calculation. To shorten notation we temporarily use units with $m=1$. First, the angular velocity $\Omega$ of fluid particles on geodesic circular orbits in the equatorial plane of the Kerr metric (1) was found, using (2):

$$
\Omega(r)=\frac{8 r^{3 / 2}}{\left((2 r+1)^{2}-a^{2}\right)^{3 / 2}+8 a r^{3 / 2}} .
$$

The angular momentum (4) reads

$$
j=-\frac{N}{D}
$$

where

$$
\begin{aligned}
D & =2 r^{5}\left(a^{2}-(2 r+1)^{2}\right)^{2} \\
& \times\left[a^{7}-3 a^{5}(1-2 r)^{2}+a^{3}\left(8 r\left(r\left(6 r^{2}+20 r+3\right)-3\right)+3\right)\right. \\
& \left.-a(2 r+1)^{2}(4(r-2) r+1)^{2}\right], \\
N & =\left(a r^{9 / 2}\left((2 r+1)^{2}-a^{2}\right)^{3 / 2}+8 a^{2} r^{6}\right) \\
& \times\left(a^{4}+a^{2}(8(r-1) r-2)-16 a r \sqrt{r\left((2 r+1)^{2}-a^{2}\right)}\right. \\
& \left.+(2 r+1)^{4}\right)\left[a^{4}+a^{2}\left(-8 r^{2}+4 r-2\right)\right. \\
& \left.-16 a r \sqrt{r\left((2 r+1)^{2}-a^{2}\right)}+(2 r+1)^{2}(4(r-2) r+1)\right] .
\end{aligned}
$$

Formulas (23) and (24) give the desired relation $j(\Omega)$ in the parametric form. In order to eliminate $r$, notice that 23 can be written as a quadratic equation

$$
\left(\frac{1}{\Omega}-a\right)^{2 / 3}=\frac{1}{4 r}\left((1+2 r)^{2}-a^{2}\right) .
$$

Denoting

$$
\xi=\left(\Omega^{-1}-a\right)^{1 / 3},
$$

solving it for $r$ and substituting the result into the formula for $j(r)$ would give, in principle, the desired result. Unfortunately, it takes form of nested radicals composed of a large number $\left(\sim 10^{3}\right)$ of terms. This is a difficult problem for nonrational values of $a$ [23]; computer algebraic systems do not help in simplifying such formulae.
On the other hand, we observe that the formula greatly simplifies for rational $a$, e.g., $a=-2 / 3$. The presence of multiple repetitions of roots of the numerator and the denominator strongly suggests the existence of a much simpler expression. A simplification of the formula for $j(\Omega)$ proceeds as follows. We define

$$
X=\sqrt{a^{2}-2 \xi^{2}+\xi^{4}}
$$

and

$$
Z=\sqrt{1-a^{2}+2 X\left(1+X-\xi^{2}\right)} .
$$

One can write the formula 24 in the form

$$
j(\xi)=-\frac{N_{1} N_{2} N_{3}}{D_{1} D_{2} D_{3} D_{4}},
$$

where

$$
\begin{aligned}
& N_{1}=a+\xi^{3}, \\
& N_{2}=\xi\left(\xi^{2}-3\right) Z^{2}+2 a\left(1+X-\xi^{2}\right) Z, \\
& N_{3}=\left(\xi^{4}+a^{2}\right) Z^{2}+2 a \xi\left(1+X-\xi^{2}\right) Z,
\end{aligned}
$$

$$
\begin{aligned}
D_{1} & =\xi^{3}, \quad D_{2}=\xi^{2}\left(\xi^{2}-3\right)^{2}-4 a^{2}, \quad D_{3}=\xi^{2}-1-X, \\
D_{4} & =1+3 X-(9+8 X) \xi^{2}+4(3+X) \xi^{4}-4 \xi^{6} \\
& +a^{2}\left(3+X-3 \xi^{2}\right) .
\end{aligned}
$$

One can easily check that $D_{3} D_{4}=-Z^{4}$, so that we get

$$
j(\xi)=\frac{\left(a+\xi^{3}\right) N_{2} N_{3}}{D_{2} \xi^{3} Z^{4}} .
$$

Now we rewrite Eq. 30 as follows:

$$
2 a\left(1+X-\xi^{2}\right) Z=N_{2}-\xi\left(\xi^{2}-3\right) Z^{2} ;
$$

by squaring the above equation we get rid of the square root in $Z$. We solve the squared equation with respect to $N_{2}$. The physical root reads

$$
N_{2}=\left[\xi\left(\xi^{2}-3\right)-2 a\right] Z^{2} .
$$

We proceed similarly with the factor $N_{3}$, obtaining the physical root:

$$
N_{3}=\left(\xi^{4}-2 a \xi+a^{2}\right) Z^{2} .
$$

On the other hand

$$
D_{2}=\left[\xi\left(\xi^{2}-3\right)-2 a\right]\left[\xi\left(\xi^{2}-3\right)+2 a\right] .
$$

After inserting (33), (34) and (35) into (32) one ends up with

$$
j(\xi)=\frac{\left(a+\xi^{3}\right)\left(\xi^{4}-2 a \xi+a^{2}\right)}{\xi^{3}\left[\xi\left(\xi^{2}-3\right)+2 a\right]} .
$$

Finally, using (26) one obtains the following formula:

$$
j(\Omega)=-\frac{1}{2} \frac{d}{d \Omega} \ln \left\{1-\left[a^{2} \Omega^{2}+3 \Omega^{\frac{2}{3}}(1-a \Omega)^{\frac{4}{3}}\right]\right\},
$$


which can be easily generalized to the case with $m=$ $w^{2} \neq 1$, by the substitution $\Omega \rightarrow w^{2} \Omega, a \rightarrow a / w^{2}, j \rightarrow$ $j / w^{2}$. We get

$$
j(\Omega)=-\frac{1}{2} \frac{d}{d \Omega} \ln \left\{1-\left[a^{2} \Omega^{2}+3 w^{\frac{4}{3}} \Omega^{\frac{2}{3}}(1-a \Omega)^{\frac{4}{3}}\right]\right\} .
$$

We shall write this expression as

$j(\Omega)=-\frac{3}{2 \kappa} \frac{d}{d \Omega} \ln \left\{1-\frac{\kappa}{3}\left[a^{2} \Omega^{2}+3 w^{\frac{4}{3}} \Omega^{\frac{2}{3}}(1-a \Omega)^{\frac{4}{3}}\right]\right\}$.

It is easy to see that for $a=0$ and $\kappa=3$ we recover the spinless rotation curve 22.

The present numerical code was also adapted to the new rotation curve. Section IV.A.5 reports results on its numerical convergence and the second example in Sec. VIII also uses the new law 38 .

We conjecture that the rotation law (39) holds not only for massless disks of dust, but also for massive toroids, and with the parameter $\kappa=3$. This hypothesis is supported by partial numerical results.

\section{TESTING THE POINCARÉ-WAVRE PROPERTY IN GENERAL-RELATIVISTIC KEPLERIAN TOROIDS}

The general relativistic and Newtonian rotations of cylindrically symmetric tori circulating around a symmetry axis differ fundamentally in one important aspect. In the Newtonian case the angular velocity $\Omega$ is a function of the geometric distance from the rotation axis only. This is a classical result of Poincaré-Wavre. Rephrasing the same in the terminology of [24, the von Zeipel cylinder of constant $\Omega$ lies at a constant geometric distance from the symmetry axis. In contrast to that, in general relativity the angular velocity of a particle of fluid within a torus becomes a function both of its circumferential distance from the rotation axis and the distance from the plane of the symmetry. Different points lying on the von Zeipel cylinder are not equidistant from the symmetry axis. This might manifest observationally through widening of spectral lines of radiation emitted by sources lying on the von Zeipel cylinder. The same would be true about characteristic radiation lines of sources located on an circumferentially-equidistant cylinder, as hinted in the $1 \mathrm{PN}$ analysis of spinless black holes [6, 7, 25, 26].

We shall discuss two solutions. One of them is a system obeying the rotation law 22 with a spin-less black hole. In the second solution the toroid rotates according to (38) (with $a=0.9$ ), while the black hole has a large spin parameter $a=0.9$. We show the dependence of the angular velocity along a cylinder having a fixed value of the circumferential radius $r_{\mathrm{C}}$.

Figure 13 corresponds to the non-spinning black hole characterized by $M_{\mathrm{BH}}=1.29$. The asymptotic mass of the system reads $M_{\mathrm{ADM}}=3.74$. The circumferential radii of the innermost and outermost points on the torus

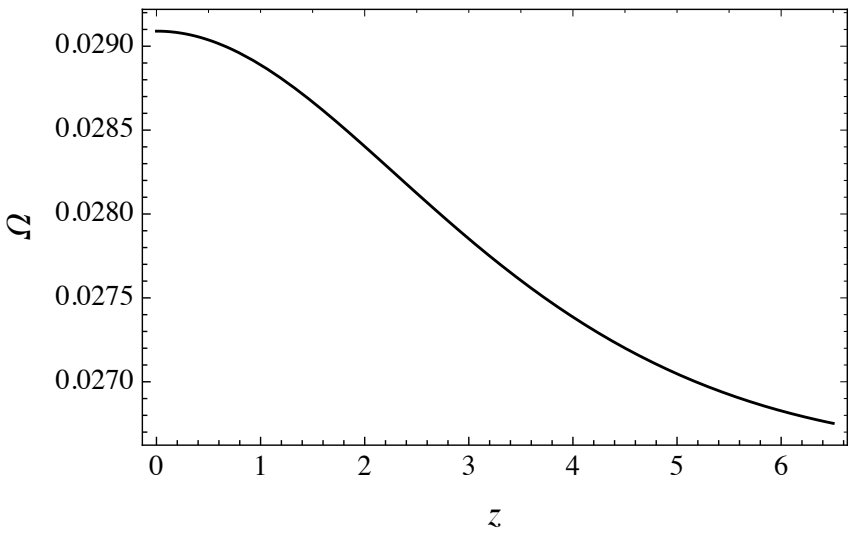

FIG. 13. Nonspinning black hole and the rotation law 22 . The innermost and outermost coordinate radii are $r_{1}=5.037$ and $r_{2}=20.09$, respectively. The coordinate $z=r \cos \theta$. The maximal mass density $\rho_{\max }=7 \times 10^{-4}$.

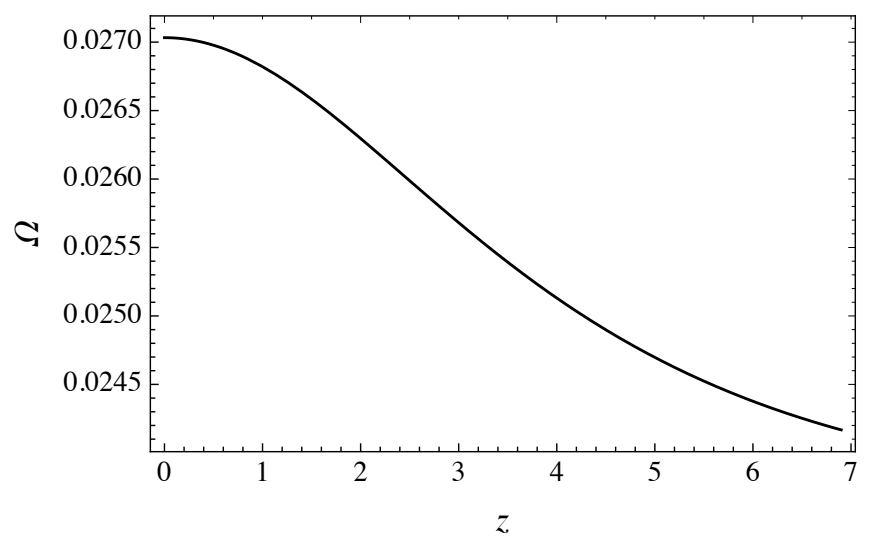

FIG. 14. Spinning black hole and the rotation law (39). The innermost and outermost coordinate radii are $r_{1}=5.027$ and $r_{2}=20.08$, respectively. The coordinate $z=r \cos \theta$. The maximal mass density $\rho_{\max }=8 \times 10^{-4}$.

are $r_{\mathrm{C}}\left(r_{1}\right)=8.01$ and $r_{\mathrm{C}}\left(r_{2}\right)=24.36$. The largest relative change of the angular velocity along a cylinder having the circumferential radius $r_{\mathrm{C}}=14.29$ exceeds $9 \%$.

Somewhat stronger effects are shown for spinning black holes. The corresponding example is shown in Fig. 14. The model is characterized by the black hole mass $M_{\mathrm{BH}}=1.033$ and its spin parameter $a=0.9$. The asymptotic mass of the system reads $M_{\mathrm{ADM}}=4.074$. The circumferential radii of the innermost and outermost points on the torus are $r_{\mathrm{C}}\left(r_{1}\right)=8.05$ and $r_{\mathrm{C}}\left(r_{2}\right)=24.79$. The largest relative change of the angular velocity along a cylinder having the circumferential radius $r_{\mathrm{C}}=15.09$ exceeds $11 \%$ (see Fig. 14.

In both cases the dependence on height is quite robust, particularly for the spinning black hole. The angular velocity drops down from the maximal value on the equator to the minimum value on the edge of the disk. Admittedly these examples have unrealistically large masses of tori. 


\section{SUMMARY}

We report in this paper results of a successful numerical implementation of a recent rotation law [6] in the full Einstein theory. This is done within the puncture framework as implemented by Shibata 9, but with several modifications.

Our numerical codes are now capable to describe a class of self-gravitating tori in the regime of strong gravity for those general-relativistic rotation curves that in the Newtonian limit yield angular velocities $\Omega \propto 1 / \varpi^{\lambda}$, with $0 \leq \lambda<1$. This class includes stationary disks in tight accretion systems with central (spinless or spinning) black holes, and it contains polytropic tori with the general relativistic Keplerian rotation, almost constant linear velocity and the constant angular momentum density.

Our numerical codes successfully passed several numerical tests. We recovered many of solutions found in [9]. Our results agree well in the post-Newtonian regime with the earlier post-Newtonian analysis (see 77, 22, 25]), but the related material will be published elsewhere.

Section VI shows profiles of several tori. It is interesting that they differ morphologically - they flatten when the rotation curve approaches the constant linear velocity, and become "fatter" for Keplerian rotation and in the case of constant angular momentum.

We have found in Sec. VII a new general-relativistic Keplerian rotation law, that generalizes the former one [6] it is expected to be more effective in the case of spinning central black holes. The present numerical code successfully operates - after adaptation - with the new rotation curve (see some results in Secs. IV.A.5 and VIII), but the investigation is still under way.
It is known that highly relativistic tori in almost Keplerian rotation can be created in the merger of compact binaries consisting of pairs of black holes or neutron stars 12 15, 27, 28, associated with the emission of gravitational waves [29]. Their formation seems to be important for understanding features of the electromagnetic radiation associated with these mergers. Our codes allow a quick manufacturing of such polytropic tori-this is a question of tens of minutes or at most hours on a standard desktop computer. They could be used as initial data for dynamic systems supplied with all required physics.

Rotating tori can exist in some active galactic nuclei. They might reveal some general-relativistic effects due to the phenomena discussed here. We have shown that the general-relativistic angular velocity is height-dependent (13); that might cause widening of spectral lines of radiation emitted by the disk (via Doppler effect) and obscure the interpretation of the rotation, especially for tight AGN systems.

Rotation curves (6) might be used for the description of rotating stars, after appropriate modification in the vicinity of the symmetry axis.

\section{ACKNOWLEDGMENTS}

This research was carried out with the supercomputer "Deszno" purchased thanks to the financial support of the European Regional Development Fund in the framework of the Polish Innovation Economy Operational Program (Contract no. POIG. 02.01.00-12-023/08). P. M. acknowledges the financial support of the Narodowe Centrum Nauki Grant No. DEC-2012/06/A/ST2/00397. M. P. acknowledges partial support from the Grant No, K/DSC/004356.
[1] E. Butterworth and I. Ipser, Astrophys. J. 200, L103 (1975).

[2] J. M. Bardeen, Astrophys. J. 162, 71 (1970).

[3] F. Galeazzi, S. Yoshida, and Y. Eriguchi, Astron. Astrophys. 541, A156 (2012).

[4] K. Uryu, A. Tsokaros, F. Galeazzi, H. Hotta, M. Sugimura, K. Taniguchi, and S. Yoshida, Phys. Rev. D 93, 044056 (2016).

[5] K. Uryu, A. Tsokaros, L. Baiotti, F. Galeazzi, K. Taniguchi, and S. Yoshida, Phys. Rev. D 96, 103011 (2017).

[6] P. Mach and E. Malec, Phys. Rev. D 91, 124053 (2015).

[7] J. Karkowski, P. Mach, E. Malec, M. Piróg, and N. Xie, Phys. Rev. D 94, 124041 (2016).

[8] J. F. G. Auchmuty and R. Beals, Arch. Ration. Mech. Anal. 43, 255 (1971).

[9] M. Shibata, Phys. Rev. D 76, 064035 (2007).

[10] I. Hachisu, Astrophys. J. Suppl. Ser. 61, 479 (1986).

[11] L. Baiotti and L. Rezzolla, Rep. Prog. Phys. 80, 096901 (2017).

[12] W. Kastaun and F. Galeazzi, Phys. Rev. D 91, 064027 (2015).
[13] L. Rezzolla and P. Kumar, Astrophys. J., 802, 95 (2015).

[14] A. Endrizzi, R. Ciolfi, B. Giacomazzo, W. Kastaun, and T. Kawamura, Classical Quantum Gravity. 33, 164001 (2016).

[15] M. Shibata, Sh. Fujibayashi, K. Hotokezaka, K. Kiuchi, K. Kyutoku, Y. Sekiguchi, and M. Tanaka, Phys. Rev. D 96, 123012 (2017).

[16] W. Kastaun, F. Galeazzi, D. Alic, L. Rezzolla, and J. A. Font, Phys. Rev. D 88, 021501 (2013).

[17] L. G. Fishbone and V. Moncrief, Astrophys. J. 207, 962(1976).

[18] S. R. Brandt and E. Seidel, Phys. Rev. D 52, 856 (1995).

[19] M. Ansorg and D. Petroff, Phys. Rev. D 72, 024019 (2005).

[20] T. Banachiewicz, Bull. Acad. Pol. Sci., Ser. Sci. Phys. Astron. 10-12, 393 (1938).

[21] E. Anderson et al., LAPACK Users' Guide (SIAM, 1999).

[22] J. Knopik, P. Mach, and E. Malec, Acta Phys. Pol. B 46, 2451 (2015).

[23] S. Landau, SIAM J. Comput. 21, 85 (1992).

[24] M. Kozlowski, M. Jaroszynski, and M. A. Abramowicz, Astron. Astrophys. 63, 209 (1978). 
[25] P. Jaranowski, P. Mach, E. Malec, and M. Piróg, Phys Rev. D 91, 024039 (2015).

[26] P. Mach, E. Malec, and M. Piróg, in The Fourteenth Marcel Grossmann Meeting on Recent Developments in Theoretical and Experimental General Relativity, Astrophysics, and Relativistic Field Theories: Proceedings of the MG14 Meeting on General Relativity University of Rome "La Sapienza", Italy, edited by M. Bianchi and
R. T. Jantzen (World Scientific, Singapore, 2017), pp. 1994-1999.

[27] F. Pannarale, A. Tonita, and L. Rezzolla, Astrophys. J. 727, 95 (2011)

[28] G. Lovelace, M. D. Duez, F. Foucart, L. E. Kidder, H. P. Pfeiffer, M. A. Scheel, and B. Szilagyi, Classcal Quantum Gravity 30, 135004 (2013).

[29] B. P. Abbott et al., Phys. Rev. Lett. 119, 161101 (2017). 